\title{
Fluctuations in serum ethanol concentration in the treatment of acute methanol poisoning: a prospective study of 21 patients
}

\author{
Sergey Zakharov ${ }^{\mathrm{a}}$, Tomas Navratil ${ }^{\mathrm{b}, \mathrm{c}}$, Tomas Salek ${ }^{\mathrm{d}}$, Ivana Kurcova ${ }^{\mathrm{e}}$, Daniela Pelclova ${ }^{\mathrm{a}}$
}

\begin{abstract}
Objective. During the 2012 outbreak of mass methanol poisonings in the Czech Republic, ethanol, in the main, was used as an antidote. The complex pharmacokinetics of ethanol made it difficult to maintain the requisite 1000-1500 $\mathrm{mg} / \mathrm{L}$ serum ethanol levels (S-EtOH). The aim of this study was to measure the fluctuations in S-EtOH during the treatment.

Methods. A prospective case series in 21 patients, median age 52 (27-79 years), 13 males and 8 females. Serum ethanol, methanol and formate were measured every 2-6 hours during the treatment. Follow-up clinical examination was carried out in 15/18 survivors.

Results. The majority of patients (17/21) were late presenters and on admission, almost half (10/21) had suffered a severe grade of intoxication according to the Poisoning Severity Score (PSS). The mean observation time was $90 \pm 20$ h. The mean period of consistent maintenance of S-EtOH within the recommended therapeutic range lasted $28 \pm 7 \%$ of the total observation time. For $29 \pm 8 \%$ of the time, S-EtOH was $>1500 \mathrm{mg} / \mathrm{L}$ with "peaks" of up to $3500 \mathrm{mg} / \mathrm{L}$. For $44 \pm 10 \%$ of the observation time, S-EtOH was $<1000 \mathrm{mg} / \mathrm{L}$. The mean duration of sub-therapeutic concentration of S-EtOH and toxic serum levels of methanol $>200 \mathrm{mg} / \mathrm{L}$ or formate $>20 \mathrm{mg} / \mathrm{L}$ lasted $20 \pm 10 \%$ and $18 \pm 11 \%$ of the time of observation, respectively. Complications occurred in 14 (67\%) of cases including significant fluctuations of S-EtOH in 9; aspiration pneumonia in 3 and delirium tremens in 2 cases. Other complications included sepsis, bleeding, acidosis rebound, intolerance and set clotting. The outcomes were: 11 survivors free of health impairment, 7 with sequelae and 3 deaths. There was no significant difference in mean duration of sub-therapeutic and supra-therapeutic concentrations of serum ethanol in patients who survived without sequelae and those with poor outcome $(P>0.05)$.

Conclusion. Administration of ethanol according to the present guidelines of the AACT/EAPCCT is effective and relatively safe in the treatment of methanol poisoning during a mass outbreak ${ }^{31}$. Physicians have to be most aware of fluctuations in serum ethanol at the end of short sessions of IHD and after changes in route from intravenous to oral. Rigorous monitoring of serum ethanol concentrations is pivotal for severely poisoned patients with PSS 3 and where there is suspected conversion of significant amounts of methanol to formic acid.
\end{abstract}

Key words: methanol poisoning, ethanol, antidote, adverse events, variations in serum levels, treatment outcome, visual sequelae, CNS sequelae

Received: September 21, 2014; Accepted with revision: February 6, 2015; Available online: March 1, 2015 http://dx.doi.org/10.5507/bp.2015.008

${ }^{a}$ Toxicological information center, Department of Occupational Medicine, $1^{\text {st }}$ Faculty of Medicine, Charles University in Prague and General University Hospital, Prague, Czech Republic

${ }^{b}$ Department of Biomimetic Electrochemistry, J. Heyrovsky Institute of Physical Chemistry of AS CR, v.v.i, Prague

Institute of Medical Biochemistry and Laboratory Diagnostics, 1 ${ }^{\text {st }}$ Faculty of Medicine, Charles University in Prague and General University Hospital, Prague

${ }^{d}$ Department of Clinical Biochemistry, Tomas Bata Regional Hospital, Zlin

${ }^{e}$ Department of Toxicology and Forensic Medicine, $1{ }^{\text {st }}$ Faculty of Medicine, Charles University in Prague and General University Hospital, Prague

Corresponding author: Sergey Zakharov, e-mail: Sergey.Zakharov@vfn.cz

\section{INTRODUCTION}

Methanol in illicit alcoholic drinks remains an important cause of outbreaks of mass poisonings throughout the world, resulting in high mortality and serious health damage $^{1-4}$. Formate anions as the products of methanol metabolism have a strong cytotoxic effect by inhibition of mitochondrial respiration ${ }^{5,6}$. The accumulation of formic acid results in metabolic acidosis, lactacidemia, visual impairment, and damage of basal ganglia ${ }^{7-10}$. Timely administration of antidotes, ethanol or fomepizole, prevents toxic metabolite formation by blocking the alcohol dehydrogenase $(\mathrm{ADH})$ enzyme $\mathrm{e}^{11-13}$.

The role of ethanol in the treatment of acute methanol poisonings is well-established ${ }^{14,15}$. Ethanol has approximately ten times higher affinity to ADH than methanol, so it blocks effectively the enzyme when its concentration in the blood serum is between $1000-1500 \mathrm{mg} / \mathrm{L}$ (22$33 \mathrm{mmol} / \mathrm{L}$ ) (ref. ${ }^{13,16}$ ). Fomepizole (4-methylpyrazole) is another effective antidote with affinity to ADH several thousand times higher than that of methanol ${ }^{17-19}$.

Some evidence exists of the superiority of fomepi- 
zole in the treatment of methanol-poisoned patients ${ }^{19,20}$. Several authors state that ethanol may cause CNS and respiratory depression, hypoglycemia during administration, and generally has a less safe side-effect profile than fomepizole ${ }^{21-24}$. Ethanol administration is associated with higher risk of medication errors and adverse reactions ${ }^{25,26}$. Higher risk of wrong dosing and adverse reactions is mainly related to the unpredictable pharmacokinetics of ethanol, especially during hemodialysis sessions ${ }^{27-29}$.

However, there are no prospective clinical studies on the consistency of maintaining of serum ethanol in recommended therapeutic concentrations with regards to the complications and adverse reactions during the therapy and the outcomes of acute methanol poisoning. In this study we report the data based on the recent mass methanol poisoning in the Czech Republic in 2012 (ref. ${ }^{30}$ ). We studied 21 methanol-poisoned patients treated with ethanol to determine how consistently serum concentration was maintained within the therapeutic range, and to study possible association between serum ethanol fluctuations and treatment outcome.

\section{MATERIAL AND METHODS}

\section{Patients and Procedures}

The study was designed as a prospective case series study. A total of 121 cases of methanol poisonings with 41 deaths occurred during the period from $3^{\text {rd }}$ September

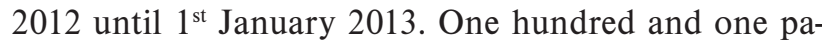
tients were treated in 30 hospitals in 11 regions of the Czech Republic. Ninety one methanol-poisoned patients were treated with antidotes. Ethanol as a single antidote was administered in 70 cases, and in a combination with fomepizole in 16 patients.

In 21 cases, the series of venous blood samples were obtained for further laboratory analysis in accordance with the study protocol described in the Laboratory investigations section. These cases were included in the prospective study and documented using a standardized admission protocol developed after the Norwegian methanol outbreak ${ }^{2}$, and the discharge reports of these patients with the results of the neurological and ophthalmologic examinations on admission, during hospitalization, and on discharge were collected and analyzed in the Czech Toxicological Information Center (TIC). Detailed medical record of the history of poisoning, the onset, and the dynamics of clinical signs and symptoms of ocular and systemic toxicity was obtained either directly from the patients or from the relatives of critically ill patients on admission, and specified retrospectively in standardized questionnaires.

On admission, the laboratory investigations included serum concentrations of methanol, ethanol, formate, lactate, electrolytes, arterial blood gases, anion and osmolal gaps, glucose, renal- and hepatic tests, complete blood count, hematocrit, and serum proteins. The diagnosis was established if (1) a history of recent ingestion of illicit spirit was available, and serum methanol concentration was more than $6.2 \mathrm{mmol} / \mathrm{L}(200 \mathrm{mg} / \mathrm{L})$, and/or an os- molal gap higher than $19 \mathrm{mOsm} /\left(\mathrm{kg} \mathrm{H}_{2} \mathrm{O}\right)$ were noted, or (2) there was a history/clinical suspicion of methanol poisoning, serum methanol detectable, and at least two of the following were present: $\mathrm{pH}$ less than 7.3 , serum bicarbonate less than $20 \mathrm{mmol} / \mathrm{L}(20 \mathrm{mEq} / \mathrm{L})$, and anion gap more than $19 \mathrm{mmol} / \mathrm{L}(19 \mathrm{mEq} / \mathrm{L})$.

In $15 / 18$ patients ( $83 \%$ of survivors included in the study) the follow-up examination was carried out three to six months after the discharge from the hospitals within the prospective study of long-term visual and CNS sequelae of acute methanol poisonings. The survivors without follow-up examination $(3 / 18)$ were excluded from the final analysis of association between the fluctuations of serum ethanol concentration and the treatment outcome (see Table 3). The clinical examination protocol included complete ocular examination and standard ophthalmic tests (visual acuity, perimeter, color vision assessment, contrast sensibility, fundus examination), optical coherence tomography (OCT) with retinal nerve fibers layer thickness evaluation, visual evoked potentials (VEP), magnetic resonance imaging of the head, neurological and neuropsychological examinations, biochemical tests (electrolytes, glucose, glycohemoglobin, albumin, pre-albumin, renal and hepatic tests, cholesterol, lipids, thyroidstimulating hormone (TSH), vitamin $\mathrm{B}_{12}$, carbohydrate deficient transferrin (CDT), complete blood count, hematocrit, ethyl glucuronide in urine), and standardized questionnaire forms (circumstances of poisoning, medical history, comorbidities, etc.).

The patients were further divided into two groups according to the outcome of treatment: group I, the patients who survived without sequelae; group II, the patients with poor outcome (survivors with visual/CNS sequelae and the patients who died). Patients were considered to have visual impairment if the symptoms of toxic neuropathy of the optic nerve were documented on admission/during hospitalization, with pathologic findings on visual acuity, visual fields, color vision, and contrast sensitivity, and persisting lesions on fundoscopy, OCT, VEP with other symptoms of visual damage were found on discharge from the hospitals and/or three to six months after the discharge. Patients were considered as having CNS sequelae of poisoning if the symmetrical necrosis and hemorrhages of basal ganglia were present on computed tomogram during the stay in hospitals and/or magnetic resonance image of the brain 3-6 months after the discharge.

\section{TREATMENT}

All patients were treated in accordance to the American Association of Clinical Toxicology and European Association of Poisons Centers and Clinical Toxicologists (AACT/EAPCCT) practice guidelines on the treatment of methanol poisoning ${ }^{31}$. Bicarbonate, $8.4 \%$ or $4.2 \%$ solution, was administered intravenously as a buffer to patients with metabolic acidosis to correct it. Ethanol was administered intravenously as $10 \%$ solution in 5\% glucose according to the following scheme: the loading dose of approximately $800 \mathrm{mg} / \mathrm{kg}$ (7.5-8.0 mL/ 
$\mathrm{kg}$ ) during $1 \mathrm{~h}$, followed by the maintenance dose 1.0-2.0 $\mathrm{mL} / \mathrm{kg} / \mathrm{h}$ or $2.5-3.0 \mathrm{~mL} / \mathrm{kg} / \mathrm{h}$ during the hemodialysis. If ethanol was administered per os, $0.7-1.0 \mathrm{~mL} / \mathrm{kg} / \mathrm{h}$ of $20 \%$ solution was generally applied in boluses each 3 hours. In several cases, the initial bolus of pre-hospital ethanol was administered per os by paramedic/medical staff of ambulance or emergency departments. Detailed information on ethanol administration is presented in Table 2.

Fomepizole (Fomepizole EUSA, EUSA Pharma, France) was applied during the hospitalization in four patients as a bolus dose of $15 \mathrm{mg} / \mathrm{kg}$ i.v. diluted in isotonic saline, and then $10 \mathrm{mg} / \mathrm{kg}$ every 12 hours in the patients without hemodialysis, and every 4 hours during hemodialysis. From the fifth dose and on, $15 \mathrm{mg} / \mathrm{kg}$ was given in order to compensate for increased metabolism. The patients treated with the combination of ethanol and fomepizole were excluded from further analysis of association between the fluctuations of serum ethanol concentration and the treatment outcome (see Table 3).

Hemodialysis was performed if the patients fulfilled any of the following criteria: serum methanol higher than $500 \mathrm{mg} / \mathrm{L}(15.6 \mathrm{mmol} / \mathrm{L})$, metabolic acidosis with a $\mathrm{pH}<7.30$, or had visual toxicity ${ }^{31}$. The mode of dialysis, intermittent hemodialysis (IHD), extended daily dialysis (EDD), or continuous veno-venous hemofiltration/hemodialysis/hemodiafiltration (CVVH/HD/HDF), was based on several factors, such as the hemodynamic stability of a patient on admission, or the severity of poisoning, but availability also played an important role: some smaller hospitals only had CVVH/HD/HDF available in the anesthesiology departments, whereas larger hospitals usually also had IHD/EDD available.

Folates were administered to substitute the inner pool of tetrahydrofolate: folinic acid (Calcium folinate Hospira, amp. $20 \mathrm{~mL}, 10 \mathrm{mg} / \mathrm{mL}$ Hospira UK Limited, Great Britain, and folic acid (Acidum folicum Léčiva, tbl. $10 \mathrm{mg}$, Zentiva, Czech Republic)). The dose of folinic acid was $50 \mathrm{mg}$, administered intravenously, every 4-6 h until methanol and formate have been eliminated. Folinic acid was diluted in $5 \%$ glucose in water and administered over 30-60 min. If folinic acid was not available, $50 \mathrm{mg}$ of folic acid in tablets were administered orally every 3-4 h. Corticosteroids were not administered to patients with visual disturbance.

\section{Laboratory Investigations}

Venous blood samples for serum ethanol, methanol and formate analysis were obtained on admission, at the start of hemodialysis, each 2-4 h during the IHD/EDD sessions or each 4-6 h during CVVHD/HDF sessions, and each 6-7 $\mathrm{h}$ after the end of dialysis. Blood samples were spun, serum separated and frozen until analyses.

Methanol was measured using gas chromatography with flame ionization detection and a direct injection with internal standard (Gas Chromatograph Chrom 5, Laboratory Instruments Prague, Czech Republic), limit of detection $1.9 \mathrm{mmol} / \mathrm{L}(60 \mathrm{mg} / \mathrm{L})$ and day-to-day coefficient of variation 2.5-5.4\%. Calibrators and controls were made by dilution of methanol p.a. (Penta, Czech Republic).
Formate was measured enzymatically on a Hitachi analyzer (Hitachi 912, Hitachi Science Systems Ltd., Japan) using formate dehydrogenase (Roche, France) and nicotinamide adenine dinucleotide (NAD) (Roche, France). Pure sodium formate (Sigma-Aldrich, USA) was used to prepare a standard of $1.1 \mathrm{mmol} / \mathrm{L}$ phosphate buffer and two control sera. Day-to-day coefficient of variation was $5.6 \%$, and the upper reference limit was $0.4 \mathrm{mmol} / \mathrm{L}$ (20 mg/L).

Serum ethanol was analyzed by gas chromatography with flame ionization detection and a direct injection with internal standard (Gas Chromatograph Chrom 5, Laboratory Instruments Prague, Czech Republic). Limit of detection is $0.9 \mathrm{mmol} / \mathrm{L}(40 \mathrm{mg} / \mathrm{L})$ and day-to-day coefficient of variation 3.8-7.1\%. Ethanol standards were purchased (Erba Lachema, Czech Republic). Osmolality was measured by freezing point depression method on a Fiske one-ten osmometer. Reference range for the osmolal gap was -9-19 mOsm/( $\left.\mathrm{kg} \mathrm{H}_{2} \mathrm{O}\right)$. The osmolal contribution from ethanol was subtracted from the measured osmolality.

\section{Calculations and Data Analysis}

The admission laboratory data, clinical symptoms, treatment measures and outcomes were compared using Two-Sample Assuming Unequal Variances (Equal Means), Two-sample F-Test for Variances, Bias test, and two-sample Kolmogorov-Smirnov test. The normality of data distribution was characterized using skewness and kurtosis tests. Data are expressed as medians and arithmetic means with either range or standard deviation or confidence interval (significance level $\alpha=0.05$ ), as appropriate. For comparison of results, common statistical tests were used ( $\mathrm{t}$-Test: Two-Sample Assuming Equal Variances, t-Test: Two-Sample Assuming Unequal Variances (Equal Means), Two-sample F-Test for Variances, Bias test, ANOVA, etc.). All statistical calculations were carried out on level of significance $\alpha=0.05$ (ref. $^{32,33}$ ). The calculations were performed using Excel 2003 (Microsoft, USA) and QC Expert software 3.1 (Trilobyte, Pardubice, Czech Republic).

\section{RESULTS}

The data of 21 patients with confirmed methanol poisoning with median age 52 (range 27-79) years, 13 males and 8 females, treated with ethanol alone (17 patients), or in a combination with fomepizole (4 patients), were analyzed. The ingested toxic spirits contained approximately 50\% methanol and 50\% ethanol in different kinds of strong alcoholic beverages (Slivovitz, Rum Tuzemak, Vodka Drak and others) with total alcohol content around $40 \%$ alcohol by volume (ABV, v/v). Only $19 \%$ of the patients were admitted within $24 \mathrm{~h}$ after methanol ingestion, $67 \%$ within $48 \mathrm{~h}$, and $14 \%$ later than $48 \mathrm{~h}$. The majority of patients $(17 / 21)$ in our study were late presenters with time to treatment more than $24 \mathrm{~h}$ from methanol ingestion; the correlation between time to treatment and the outcome was not significant $(\mathrm{r}=0.050, P=0.829)$. 


\section{Admission data, clinical features, and treatment}

The laboratory data and clinical features on admission are shown in Table 1. Five patients had detectable ethanol before hospital treatment with antidote. Two of them selfadministered ethanol shortly before the hospitalization; in further two cases bolus of ethanol was administered by the medical staff of emergency departments of smaller local hospitals before the transfer to the hospitals with ICU and dialyzing facilities available; in one case the origin of positive serum ethanol on admission was related to the recent toxic spirits ingestion.

Among the symptomatic patients, the most frequent clinical signs were gastrointestinal disturbances (nausea, upset stomach, vomiting) in 11/21 (52\%) cases and dyspnoea in $8 / 21$ (38\%) cases; features of visual toxicity (blurry or cloudy vision, central visual field defects, and alterations in light, color and depth perception) and coma were both found in 6/21 (29\%) cases.

The treatment measures are shown in Table 2.

Almost half of the patients (10/21) suffered severe poisoning with grade 3 of poisoning severity score (PSS) on admission; of them, 8 patients were intubated and were administered vasopressors/inotropes to support hemodynamics. The correlation between PSS and outcome was not significant $(\mathrm{r}=0.383, P=0.086)$. The treatment of the patients included alkalization in $14 / 21$ (67\%) cases. Folates were administered in 10/21 (48\%) subjects (folinic acid in 4 patients, folic acid in 6 patients).

Hemodialysis was applied in $18 / 21$ (86\%) cases; in 4 of them fomepizole was administered during the hemodialysis sessions. The correlation between the use of hemodialysis and outcome was not significant $(r=0.156$, $P=0.500)$.

The median length of stay on intensive care unit was 4 days (range 1-23 days).

\section{Ethanol administration, adverse reactions, and complications}

Initial bolus of ethanol was administered per os in $6 / 21(29 \%)$ patients on admission. Of them, in 4 cases with Glasgow coma scale $\leq 10$ ethanol was administered through the nasogastric tube. The initial oral dose differed from 47 to $632 \mathrm{mg} / \mathrm{kg}$ of absolute ethanol. In 4 cases, $40 \%$ solution of ethanol was administered, and $20 \%$ ethanol solution was given in two other cases. No adverse reactions were observed during the oral administration of initial bolus of ethanol.

During the hospitalization, ethanol was administered intravenously in 19/21 (91\%) patients with the standard maintenance dose of $1.0-2.0 \mathrm{~mL} / \mathrm{kg} / \mathrm{h}$ of $10 \%$ solution, which was increased to $2.5-3.0 \mathrm{~mL} / \mathrm{kg} / \mathrm{h}$ during the hemodialysis sessions. The initial loading dose of 7.5-8.0 mL/kg was administered intravenously in 14/21 (74\%) patients.

In two cases, ethanol was administered per os only. In one of them, four doses of fomepizole were followed by further administration of $20 \%$ ethanol after the serum methanol level decreased below $300 \mathrm{mg} / \mathrm{L}$ and the metabolic acidosis was corrected.

In 6/19 (32\%) patients the intravenous administration of ethanol was followed by per os administration of $20 \%$ solution of ethanol. All these patients had been dialyzed and had serum methanol below $200 \mathrm{mg} / \mathrm{L}$ and normal blood $\mathrm{pH}$ to the moment of change of the route of ethanol administration.

Complications during treatment occurred in 14/21 (67\%) cases. The most frequent complication was significant fluctuation of serum ethanol in 9/21 (43\%) cases, with episodes of serum ethanol $\geq 65.1 \mathrm{mmol} / \mathrm{L}$ (3000 $\mathrm{mg} / \mathrm{L}$ ) observed in 4 cases. Aspiration pneumonia occurred in three cases and delirium tremens developed after the termination of ethanol administration in two cases. Other complications included sepsis, bleeding due to heparinization during the hemodialysis session, rebound of metabolic acidosis, agitation, and dialysis set clotting. In one case, the oral administration of ethanol was stopped due to intolerance and was followed by the intravenous administration.

\section{Consistency of the therapeutic serum levels of ethanol and the outcomes of treatment}

The mean time of monitoring of serum ethanol concentrations in our study was $90 \pm 20$ hours, and the mean period of consistent maintenance of serum ethanol level within the therapeutic range of 22-33 mmol/L (1000-1500 $\mathrm{mg} / \mathrm{L}$ ) lasted $28 \pm 7 \%$ of the time of observation. The mean duration of periods with supra-therapeutic serum ethanol levels was $29 \pm 8 \%$, with the episodes of "peak" serum concentration of ethanol as high as $76 \mathrm{mmol} / \mathrm{L}$, or 3500 $\mathrm{mg} / \mathrm{L}$ (see Table 3 ). On the other hand, during $44 \pm 10 \%$ of the time of observation serum ethanol levels were subtherapeutic, below $22 \mathrm{mmol} / \mathrm{L}$ ( $1000 \mathrm{mg} / \mathrm{L})$. The "unprotected" periods, when serum methanol levels were higher than $6.2 \mathrm{mmol} / \mathrm{L}(200 \mathrm{mg} / \mathrm{L})$, or serum formate levels were higher than $0.4 \mathrm{mmol} / \mathrm{L}(20 \mathrm{mg} / \mathrm{L})$ and serum ethanol concentrations were sub-therapeutic, lasted $20 \pm 10 \%$ and $18 \pm 11 \%$ of the time of observation, respectively.

The correlation between the severity of poisoning and the duration of periods with sub-therapeutic or supra-therapeutic serum ethanol concentrations was not significant ( $\mathrm{r}=0.251, P=0.273$, and $\mathrm{r}=-0.280, P=0.219$, respectively). The highest and the lowest serum ethanol levels registered in the same patients during the observation time differed significantly (Fig. 1).

Nevertheless, the correlation between the severity of poisoning and the duration of "unprotected" periods with toxic levels of serum methanol was significant $(r=0.486$, $P=0.03$ ). In the most severely poisoned patients the duration of these periods was longer. However, the correlation between PSS and the duration of "unprotected" periods with toxic levels of serum formate was not significant ( $\mathrm{r}=$ $0.363, P=0.139)$.

The episodes of significant decrease of serum ethanol concentration under the therapeutic level were observed in 7 cases at the end of short sessions of IHD. In 5 of them, serum methanol and formate concentrations remained high after the first 2- or 3-hour session of IHD, requiring several (2-4) additional sessions of hemodialysis during the following days.

The episodes of extreme fluctuations of serum ethanol 


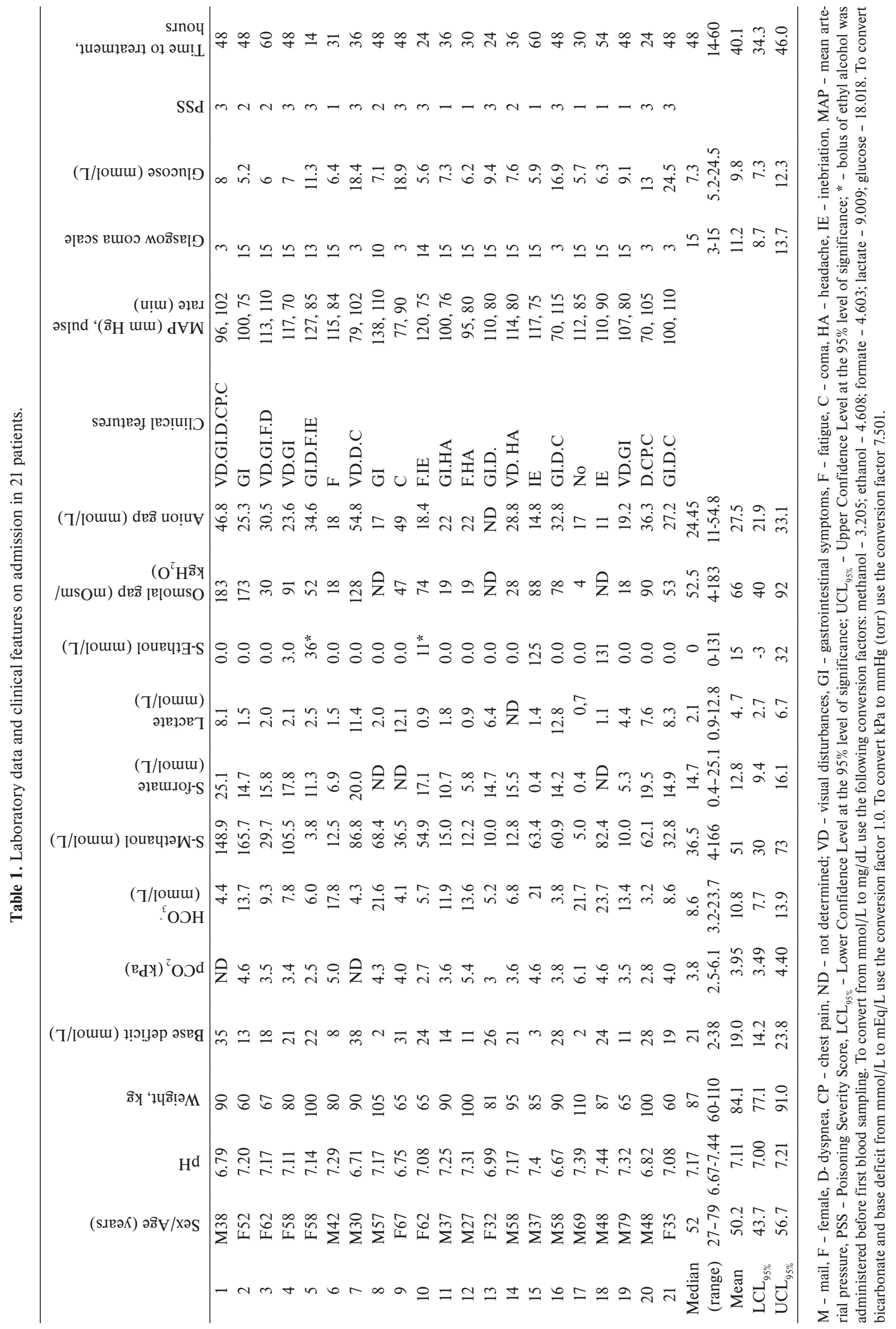




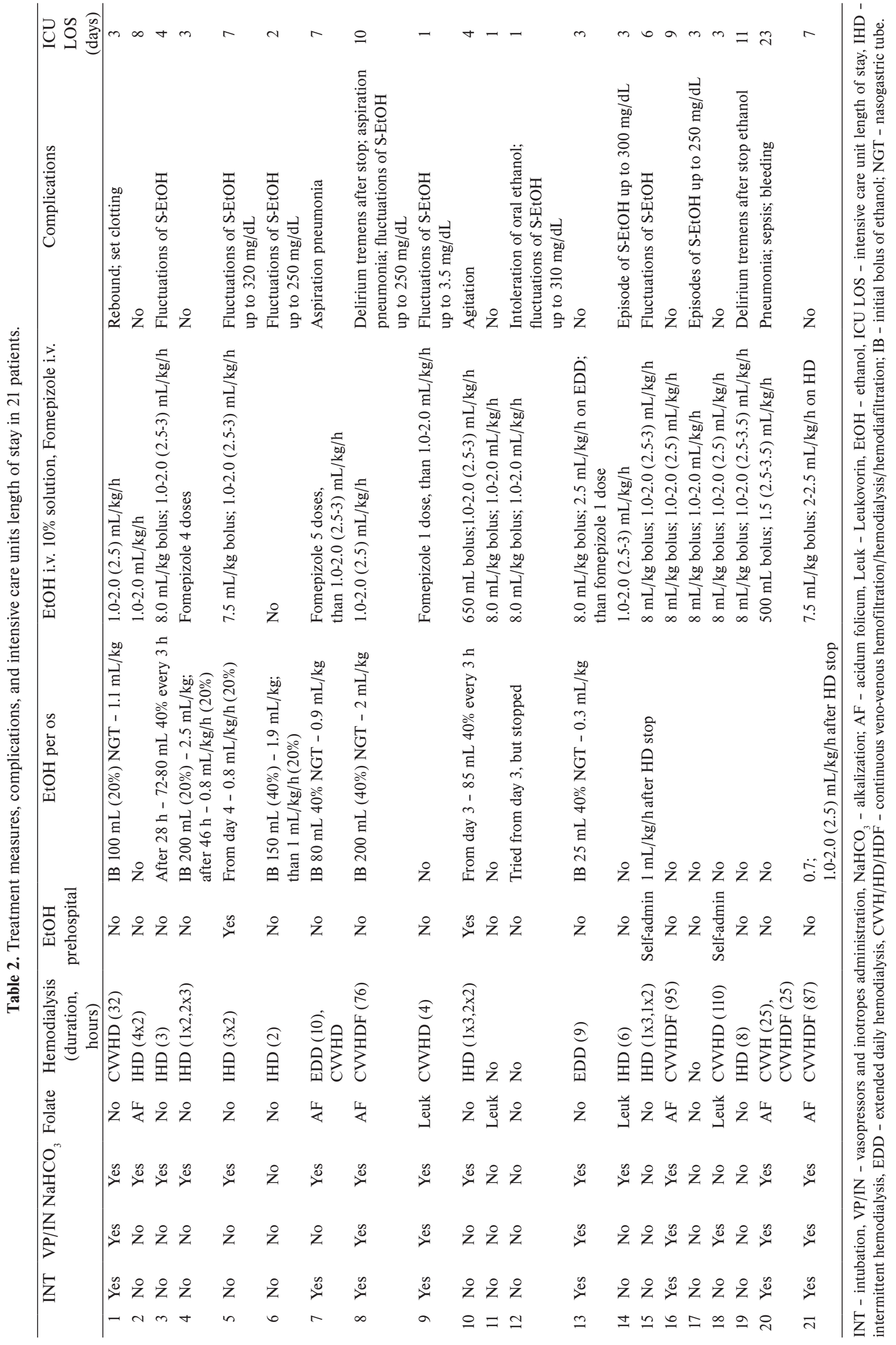




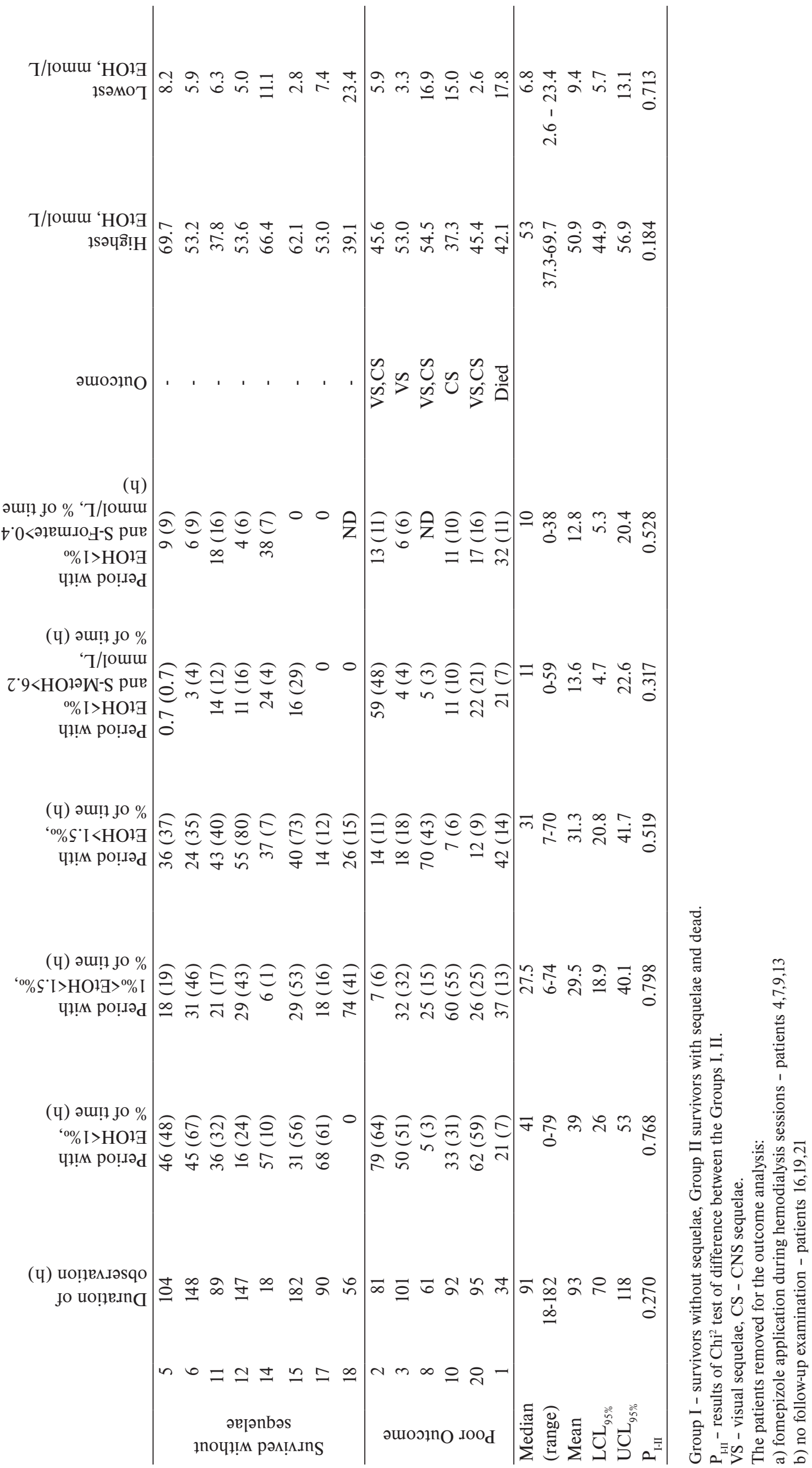




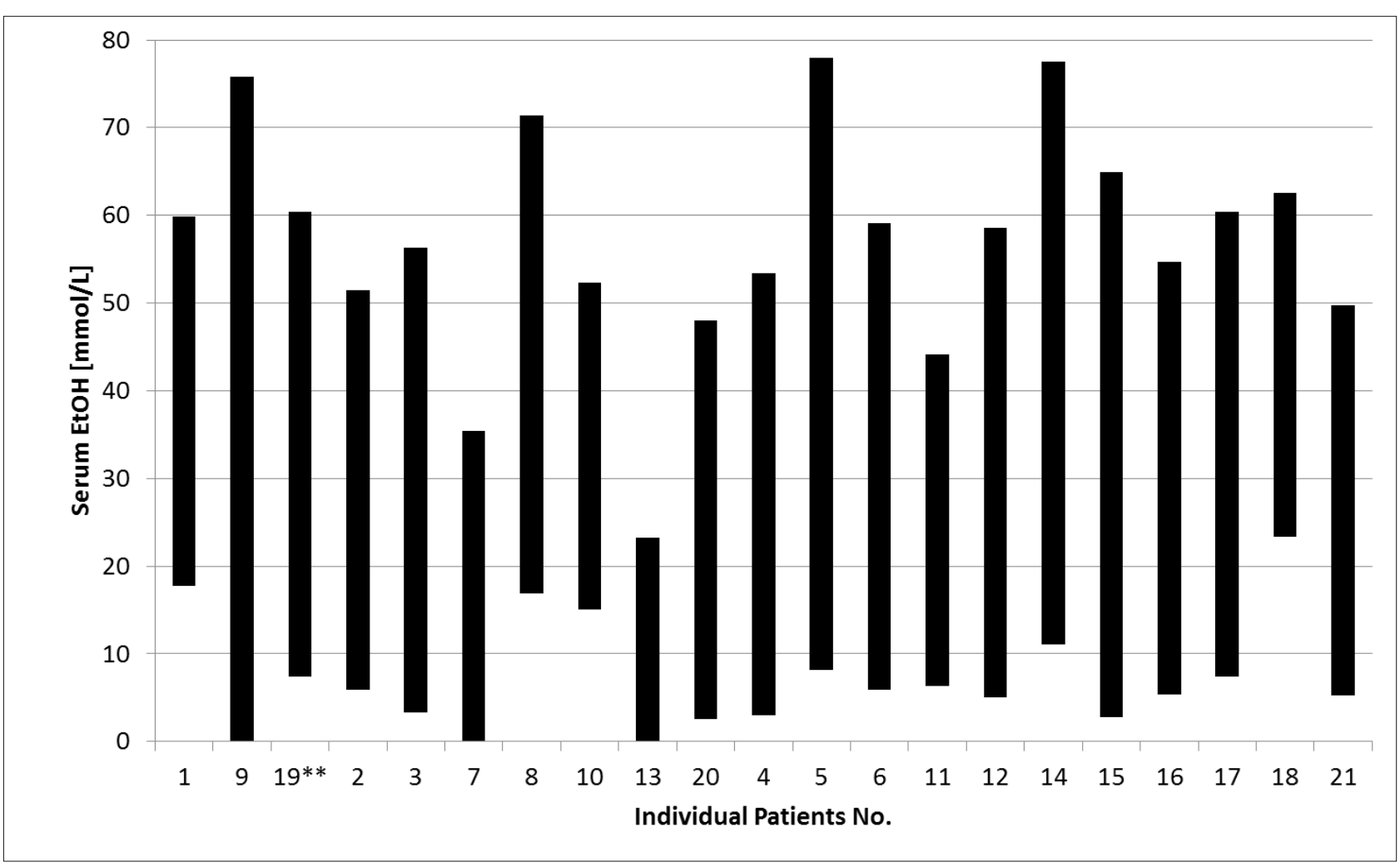

Fig. 1. Highest and lowest serum ethanol levels measured for each patient. Upper-end of boxes present the highest serum ethanol concentrations measured during the observation period; lower-end of boxes present the lowest serum ethanol concentrations measured during the observation period.

Group I - survivors without sequelae (patients \#4 - \#21), Group II - patients with poor outcome (\#1 - \#19 died; \#2 - \#20 - survived with sequelae). ${ }^{* *}$ - the patient died in hospital from infectious complications one month after the methanol poisoning.

levels were observed in 7 other cases after the change of route of administration from intravenous to oral. In these cases, no acidosis rebound was observed, serum formate remained negative, and serum methanol was already $<6.2$ $\mathrm{mmol} / \mathrm{L}(200 \mathrm{mg} / \mathrm{L})$ to the moment of the change of route of administration.

The outcomes of treatment were: 11 survivors without health impairment and 10 patients with poor outcome (7 survivors with sequelae and 3 deaths). Of 21 patients, the patients with fomepizole administration during hemodialysis sessions ( 4 cases) and the patients without followup examinations ( 3 cases), were excluded from the further analysis of possible association between serum ethanol fluctuations and the outcomes of treatment (see Table 3).

The association between the outcomes of treatment and the duration of periods with sub-therapeutic or supra-therapeutic serum ethanol concentrations was not significant $(\mathrm{r}=0.199, P=0.387$, and $\mathrm{r}=-0.157, P=0.498$, respectively). Further, the association between the outcomes and the duration of "unprotected" periods with sub-therapeutic serum ethanol concentrations and toxic serum levels of methanol or formate was not significant either $(\mathrm{r}=0.433, P=0.056$, and $\mathrm{r}=0.371, P=0.129$, respectively).

The difference in mean duration of periods with subtherapeutic, as well as with supra-therapeutic serum concentrations of ethanol was not significant between the two group of patients, the survivors without sequelae and the patients with poor outcome (see Table 3).

\section{DISCUSSION}

Methanol poisonings have a high mortality and incidence of long-term health sequelae in spite of a complex and resource-consuming treatment ${ }^{31}$. Fomepizole administration as a first-choice antidote is a well-established treatment in patients with acute methanol poisoning ${ }^{19-22}$. However, high cost and limited availability of fomepizole make the issue of ethanol administration as a cheaper and more available antidote still actual, especially during outbreaks of mass methanol poisonings. The general principle is that an antidote should be administered as soon as possible after methanol ingestion, and ethanol is often a single choice "at hand" in mass methanol outbreaks.

Nevertheless, administration of ethanol as an antidote can be associated with some pitfalls, mainly with errors of dosing and adverse reactions ${ }^{25,26}$. It can cause CNS and respiratory depression in the cases of overdose, altering the patients' ability to hyperventilate and thus removing this important compensating mechanism of metabolic acidosi $^{34}$. On the other hand, low sub-therapeutic serum concentrations of ethanol might be insufficient for the 
complete block of ADH enzyme and prevention of further metabolism of methanol to toxic formic acid. According to some authors, serum ethanol levels are consistently maintained $\geq 22 \mathrm{mmol} / \mathrm{L}(1000 \mathrm{mg} / \mathrm{L})$ in $11.7 \%$ of the patients treated with ethanol administration only ${ }^{35}$.

The complex pharmacokinetics of ethanol during the therapy makes it rather difficult to adapt the dosage and maintain the therapeutic range of its serum concentration consistently. However, in our study despite the wide fluctuations in serum ethanol concentration during the treatment, we found no association between serum ethanol fluctuations and treatment outcome. The results of our study support the findings of another study by Roy et al. in children with methanol poisoning treated with ethanol ${ }^{36}$.

Despite the wide variation in ethanol concentration during the time of observation, the total duration of "unprotected" periods with sub-therapeutic serum concentrations of ethanol and toxic serum methanol or formate levels was approximately $20 \%$ of the time of observation only. Therefore, for most of the time of observation, the patients were sufficiently protected by blocking the $\mathrm{ADH}$ enzyme and preventing further metabolism of methanol to formic acid.

The episodes of significant decrease of serum ethanol were generally observed at the end and immediately after the termination of short sessions of IHD, indicating that most of ethanol administered as an antidote was effectively dialyzed. However, serum levels of methanol and formate decreased significantly too, therefore the remaining sub-therapeutical concentrations of ethanol were apparently sufficient to block the ADH enzyme still effectively. The target level of serum ethanol of $22 \mathrm{mmol} / \mathrm{L}$ $(1000 \mathrm{mg} / \mathrm{L})$ is not an evidence-based one, and the therapeutic level may actually be lower ${ }^{36}$.

Nevertheless, it has to be emphasized that in 3 of 7 cases, where the short sessions of IHD lasting 2-3 h were applied, the patients had visual and CNS impairment 3-6 months after discharge. In another case, health sequelae could have been underdiagnosed, because the patient had $\mathrm{pH} 7.11$ on admission, and the follow-up examination was not carried out. In the last 3 cases, the patients with short sessions of IHD survived without sequelae, but their serum formate levels on admission were low. Short sessions of IHD lasting 2-3 h are generally insufficient to eliminate serum formate, as well as serum methanol, in high concentrations ${ }^{37}$.

The significant fluctuations in serum ethanol concentrations were associated with the change of route of administration from the intravenous to the oral one. In these cases, the serum methanol level was already low, serum formate was negative, and arterial blood $\mathrm{pH}$ was normal to the moment of the change of route of ethanol administration. Therefore, the fluctuations of serum ethanol in these cases had minimal negative impact on the outcome due to preceding intensive treatment and elimination of most of the toxic agents.

In one case (patient \#6) the ethanol was administered per os only during the treatment: this patient had low serum methanol and formate concentrations on admission, and short session of IHD was performed to rapidly eliminate most of the toxic agents. Generally, the intravenous route of ethanol administration should be applied in the patients with acute methanol poisoning; the oral administration should be reserved for the prehospital "first aid" or for the cases when the intravenous administration is impossible due to objective reasons.

Therefore, physicians have to be most aware of the fluctuations of serum ethanol at the end of short sessions of IHD and after the change of route of ethanol administration from the intravenous to the oral one. Thus, thorough monitoring of ethanol concentrations is recommended here. Rigorous monitoring of the serum ethanol concentrations is pivotal for the severely poisoned patients with PSS 3 and for patients where it is suspected that significant amounts of methanol will be converted to formic acid. In our study, in these patients the relative duration of "unprotected" periods with sub-therapeutic serum ethanol concentrations and toxic serum levels of methanol was longer, increasing the possibility of deterioration of their state by further accumulation of formic acid.

In this study we found no adverse reactions which could be directly related to the administration of ethanol, except the fluctuations of serum ethanol levels. No features of severe CNS depression (coma), respiratory depression, or hypoglycemia caused by high concentrations of ethanol were registered. No patients needed intubation due to ethanol administration; all the patients with intubation either were admitted to the hospitals in a coma, or were analgosedated due to other severe conditions on admission (agitation, severe dyspnea, confusion). No cases of seizures, hypotension, bradycardia, and renal or hepatic failure due to ethanol administration were observed. The single case of the rebound of metabolic acidosis was caused by discontinuation of CVVHD due to the set clotting. Two cases of delirium tremens were caused by discontinuation of ethanol intake after stopping the antidote administration in the patients with the history of chronic alcohol abuse (daily consumption of large amounts of distillates). Other complications were caused by infectious agents and by heparinization during the hemodialysis.

Two of three patients who died had severe metabolic acidosis on admission with extremely low arterial blood $\mathrm{pH}$ and bicarbonate. One of them died due to fulminant brain edema that developed 3.5 hours after the start of CVVHD. The last 79-year-old patient died in hospital due to infectious complications one month after methanol ingestion. This patient had low serum methanol and formate on admission, arterial blood $\mathrm{pH}>7.3$, and only minor degree of methanol poisoning. The patients who survived with visual and/or CNS sequelae were generally more acidotic on admission and had higher serum methanol and formate levels than those who survived without sequelae. 


\section{LIMITATIONS}

The study had some principal limitations, because it was neither a randomized controlled trial, nor a cohort trial. It was a case series, in which the patients were not uniform; their reporting of time of ingestions and doses might not be accurate. Other factors that may have confounded the results included the co-ingestion of different types and quantities of concomitant alcoholic beverages, different temporal patterns of toxic alcohol ingestion, comorbidity, etc. Many of these factors could be established with some degree of approximation only.

Despite these limitations and confounding factors, this is to date the most comprehensive prospective study ever presented about the fluctuations in the serum ethanol concentrations in patients during the application of ethanol as an antidote in a mass methanol outbreak. The essential clinical and laboratory data on admission were collected during the hospitalization using standardized forms distributed to the hospitals by the TIC during the first weeks of the outbreak. Further, this is the first-ever study which included the follow-up examination in more than $80 \%$ of the patients who survived the poisoning 3-6 months after discharge using advanced technologies to identify and characterize the long-term visual and CNS sequelae of methanol poisonings. Most of the patients who survived were seen within the follow-up examination at the same academic medical facility according to the standardized protocol including complete ophthalmologic and neurological examinations, as well as biochemical and toxicological tests to limit the influence of other confounders.

\section{CONCLUSIONS}

Administration of ethanol according to the present guidelines of the AACT/EAPCCT is effective and relatively safe in the treatment of methanol poisonings during a mass methanol outbreak ${ }^{31}$. Physicians have to be most aware of the fluctuations of serum ethanol at the end of short sessions of IHD and after the change of route of ethanol administration from the intravenous to the oral one. Thus, thorough monitoring of ethanol concentrations is recommended here. Rigorous monitoring of the serum ethanol concentrations is pivotal for the severely poisoned patients with PSS 3 and for patients where it is suspected that significant amounts of methanol will be converted to formic acid. Despite the difficult pharmacokinetics and significant variations in serum ethanol concentrations during the treatment, we found no association between serum ethanol fluctuations and treatment outcomes. No adverse reactions were observed which could be directly related to the administration of ethanol. Given a general principle that an antidote should be administered as soon as possible after methanol ingestion, and the given the general availability of ethanol, both antidotes, fomepizole and ethanol, may be considered for ADH blockade during mass methanol outbreaks without limitations.
Acknowledgement: Supported with the Projects of the Charles University in Prague P25/1LF/2, P26/LF1/4, and P28/1LF/6, the Project of the Ministry of Health of the Czech Republic 12/14/NAP, and EU Project "Materialtechnical Research Base for the Diagnostics and Treatment of Environmentally-caused and Oncological Disorders and their Risks, in the General University Hospital in Prague" (reg. No. CZ.2.16/3.1.00/24.12). The study was approved by the General University Hospital Ethics Committee in Prague, Czech Republic.

Author contributions: SZ: data collection, data analysis, data interpretation, manuscript writing; TN: figures, statistical analysis; TS, IK: data collection, data analysis; DP: literature search, study design, critical review of the manuscript.

Conflict of interest statement: The authors declare that there are no conflicts of interest regarding the publication of this article.

\section{REFERENCES}

1. Paasma R, Hovda, KE, Tikkerberi, A, Jacobsen D. Methanol mass poisoning in Estonia: outbreak in 154 patients. Clin Toxicol 2007;45:1527

2. Hovda KE, Hunderi OH, Tafjord AB, Dunlop O, Rudberg N, Jacobsen D. Methanol outbreak in Norway 2002-2004: epidemiology, clinical features and prognostic signs. J Intern Med 2005;258:181-90.

3. Bezdicek O, Klempir J, Liskova I, Michalec J, Vaneckova M, Seidl Z, JanikovaB, Miovsky M, Hubacek JA, DiblikP, Kuthan P, Pilin A, Kurcova I, Fenclova Z, Petrik V, Navratil T, Pelclova D, Ruzicka E, Zakharov S. Sequelae of methanol poisoning for cognition. Cesk Slov Neurol N 2014;77/110:320-5

4. Zakharov S, NurievaO, Navratil T, Diblik P, Kuthan P, Pelclova D. Acute methanol poisonings: Folates administration and visual sequelae. J of Appl Biomed 2014;12:309-16.

5. Liesivuori J, Savolainen H. Methanol and Formic-Acid Toxicity Biochemical-Mechanisms. Pharmacology \& Toxicology 1991;69:15763.

6. Hovda KE, Hunderi OH, Rudberg N, Froyshov S, Jacobsen D. Anion and osmolal gaps in the diagnosis of methanol poisoning: clinical study in 28 patients. Intensive Care Med 2004;30:1842-6.

7. Zakharov S, Navratil T, Pelclova D. Analysis of serum anion gap and osmolal gap in diagnosis and prognosis of acute methanol poisoning: clinical study in 86 patients. Monatsh Chem Chem Mon 2015: doi: 10.1007/s00706-014-1374-7.

8. Zakharov S, Kurcova I, Navratil T, Salek T, Pelclova D. Is the measurement of serum formate concentration useful in the diagnostics of acute methanol poisoning? Prospective study in 38 patients. Basic Clin Pharmacol Toxicol 2015: doi: 10.1111/bcpt.12338.

9. Vaneckova M, Zakharov S, Klempir J, Ruzicka E, Bezdicek O, Liskova I, Diblik P, Miovsky M, Hubacek JA, Urban P, Ridzon P, Pelclova D, Burgetova A, Masek M, Seidl Z. Methanol Intoxication on Magnetic Resonance Imaging - Case Reports. Cesk Slov Neurol N 2014;77:2359 .

10. Osterloh JD, Pond SM, Grady S, Becker CE. Serum Formate Concentrations in Methanol Intoxication as a Criterion for Hemodialysis. Ann Intern Med 1986;104:200-3.

11. Hubacek JA, Pelclova D, Seidl Z, Vaneckova M, Klempir J, Ruzicka E, Ridzon P, Urban P, Fenclova Z, Petrik V, Diblik P, Kuthan P, Miovsky M, Janikova B, Adamkova V, Zakharov S. Rare Alleles within the CYP2E1 (MEOS System) could be associated with better short-term Health Outcome after Acute Methanol Poisoning. Basic Clin Pharmacol Toxicol 2015;116(2):168-72. doi: 10.1111/bcpt.12310.

12. Megarbane B, Borron SW, Baud FJ. Current recommendations for treatment of severe toxic alcohol poisonings. Intensive Care Med 2005;31:189-95.

13. Zakharov S, Navratil T, Pelclova D. Fomepizole in treatment of acute methanol poisonings: experience from the Czech mass methanol 
outbreak 2012-2013. Biomed Pap Med Fac Univ Palacky Olomouc Czech Repub 2014;158:641-9.

14. Bergeron R, Cardinal J, Geadah D. Prevention of Methanol Toxicity by Ethanol Therapy. N Engl J Med 1982;307:1528.

15. Hantson $P$, Wittebole $X$, Haufroid V. Ethanol therapy for methanol poisoning: duration and problems. Eur J Emerg Med 2002;9:278-9.

16. Jacobsen D, Jansen H, Wiik-Larsen E, Halvorsen S. Studies on methanol poisoning. Acta Med Scand 1982;212:5-10.

17. Burns MJ, Graudins A, Aaron CK, McMartin K, Brent J. Treatment of methanol poisoning with intravenous 4-methylpyrazole. Ann Emerg Med 1997;30:829-32.

18. Bekka R, Borron SW, Astier A, Sandouk P, Bismuth C, Baud FJ. Treatment of methanol and isopropanol poisoning with intravenous fomepizole. J Toxicol-Clin Toxicol 2001;39:59-67.

19. Brent J, McMartin K, Phillips S, Aaron C, Kuliq K. Fomepizole for the treatment of methanol poisoning. N Engl J Med 2001;344:424-9.

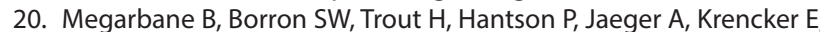
Bismuth C, Braud FJ. Treatment of acute methanol poisoning with fomepizole. Intensive Care Med 2001;27:1370-8.

21. Brent J. Fomepizole for ethylene glycol and methanol poisoning. $N$ Engl J Med 2009:360:2216-23.

22. Brent J. Fomepizole for the treatment of pediatric ethylene and diethylene glycol, butoxyethanol, and methanol poisonings. Clin Toxicol 2010;48:401-6.

23. Hovda KE, Jacobsen D. Expert opinion: fomepizole may ameliorate the need for hemodialysis in methanol poisoning. Human \& Experimental Toxicology 2008;27:539-46.

24. Borron SW, Megarbane B, Baud, FJ. Fomepizole in treatment of uncomplicated ethylene glycol poisoning. Lancet 1999;354:831.

25. Lepik KJ, Levy AR, Sobolev BG, et al. Adverse drug events associated with the antidotes for methanol and ethylene glycol poisoning: a comparison of ethanol and fomepizole. Ann Emerg Med 2009;53:439-50.

26. Lepik KJ, Sobolev BG, Levy AR, Purssell RA, Dewitt CR, Erhardt GD, Baker JL, Kennedy JR, Daws DE. Medication errors associated with the use of ethanol and fomepizole as antidotes for methanol and ethylene glycol poisoning. Clin Toxicol 2011;49:391-401.

27. McCoy HG, Cipolle RJ, Ehlers SM, Sawchuk RJ, Zaske DE. Severe methanol poisoning - application of a pharmacokinetic model for ethanol therapy and hemodialysis. Am J Med 1979;67:804-7.

28. Hantson P, Haufroid V, Wallemacq P. Formate kinetics in methanol poisoning. Human \& Experimental Toxicology 2005;24:55-9.

29. Wilkinson PK, Sedman AJ, Sakmar E, Earhart RH, Weidler DJ, Wagner JG. Blood ethanol concentrations during and following constant-rate intravenous infusion of alcohol. Clin Pharmacol Ther 1976;19:213-23.

30. Zakharov S, Pelclova D, Urban $P$, Navratil T, Diblik $P$, Kuthan $P$ Hubacek JA, Miovsky M, Klempir J, Vaneckova M, Seidl Z, Pilin A, Fenclova Z, Petrik V, Kotikova K, Nurieva O, Ridzon P, Hovda KE. Czech Mass Methanol Outbreak 2012: Epidemiology, Challenges and Clinical Features. Clin Toxicol 2014;52:1013-24.

31. Barceloux DG, Bond GR, Krenzelok EP, Cooper H, Vale JA. American Academy of Clinical Toxicology practice guidelines on the treatment of methanol poisoning. J Toxicol-Clin Toxicol 2002;40:415-46.

32. Meloun M, Militky J, Forina M. Chemometrics for Analytical Chemistry, Volume 1: PC-Aided Statistical Data Analysis, Volume 2: PC-Aided Regression and Related Methods, Chichester: Ellis Horwood, 1992.

33. Cizkova P, Navratil T, Sestakova I, Yosypchuk B. Verification of applicability of mercury meniscus modified silver solid amalgam electrode for determination of heavy metals in plant matrices. Electroanalysis 2007:19:161-71.

34. Paasma R, Hovda KE, Hassanian-Moghaddam H, Brahmi N, Afshari $\mathrm{R}$, Sandvik $\mathrm{L}$, Jacobsen D. Risk factors related to poor outcome after methanol poisoning and the relation between outcome and antidotes-a multicenter study. Clin Toxicol 2012;50:823-31.

35. Oker EE, Kanji SA, Aks S, Fischbein C. Can health care workers effectively administer ethanol drips? Acad Emerg Med 2001;8:443.

36. Roy M, Bailey B, Chalut D, Senecal PE, Gaudreault P. What are the adverse effects of ethanol used as an antidote in the treatment of suspected methanol poisoning in children? J Toxicol-Clin Toxicol 2003;41:155-61.

37. Zakharov S, Pelclova D, Navratil T, Belacek J, Kurcova I, Komzak O, Salek T, Latta J, Turek R, Bocek R, Kucera C, Hubacek JA, Fenclova Z, Petrik V, Cermak M, Hovda KE Methanol and formate elimination half-life during treatment for methanol poisoning: intermittent haemodialysis vs. continuous haemodialysis/haemodiafiltration. Kidney Int 2014:86:199-207. 